\title{
氧化铝气凝胶的合成与性能
}

\author{
杨景锋 ${ }^{1,2}$, 王齐华 ${ }^{1}$, 王廷梅 ${ }^{1}$ \\ (1. 中国科学院 兰州化学物理研究所, 固体润滑国家重点实验室, 兰州 730000; 2. 中国科学院大学, 北京 100049)
}

摘 要: 氧化铝气凝胶具有大比表面积、高孔隙率以及比氧化硅气凝胶更优异的热稳定性, 在高温隔热和催化领域 有广阔的应用前景, 但其复杂的合成工艺和较低的强度限制了其应用。本文首先介绍氧化铝气凝胶合成工艺, 指出 溶胶-凝胶过程中存在的问题, 不同干燥方式的特点以及最新发展的干燥工艺; 然后介绍了近年来氧化铝气凝胶在 比表面积、热稳定性、强度、导热系数等性能改善方面所取得的代表性研究成果; 最后介绍了氧化铝气凝胶在高温 催化、隔热领域的应用, 并展望了未来氧化铝气凝胶的发展方向以及应关注的问题。

关 键 词: 氧化铝气凝胶; 合成; 性能; 比表面积; 热稳定性; 综述

中图分类号: TB332; O648 文献标识码: A

\section{Synthesis and Property of Alumina Aerogel}

\author{
YANG Jing-Feng $^{1,2}$, WANG Qi-Hua ${ }^{1}$, WANG Ting-Mei ${ }^{1}$
}

(1. State Key Laboratory of Solid Lubrication, Lanzhou Institute of Chemical Physics, Chinese Academy of Sciences, Lanzhou 730000, China; 2. University of Chinese Academy of Sciences, Beijing 100049, China)

\begin{abstract}
Alumina aerogel has wide application prospect in high temperature thermal insulation and catalytic fields due to its high specific surface area, high porosity, and better thermal stability than silica aerogel. However, the complicated synthetic process and the low strength of alumina aerogel restrict their application. In this review, the synthetic process of alumina aerogel is firstly introduced. Some problems existed in the Sol-Gel process, the characteristics of various drying methods, and the latest drying methods developed by researchers are pointed out. Then, some representation achievements in the performance improvements on specific surface area, thermal stability, thermal conductivity and compressive strength of alumina aerogel in recent years are summarized. Finally, the development and application of alumina aerogel in high temperature catalytic and thermal insulation fields are introduced, their development tendency is prospected, and some issues on the study of alumina aerogel are highlighted.
\end{abstract}

Key words: alumina aerogel; synthesis; property; specific surface area; thermal stability; review

氧化铝气凝胶具有低密度、低导热系数、大比 表面积和高孔隙率等特点, 还具有优异的热稳定 性 ${ }^{[1-3]}$ 。氧化铝气凝胶的 $\gamma$ 相具有大的比表面积和宽 的晶相范围, 可以稳定到 $900 \sim 1000{ }^{\circ} \mathrm{C}^{[4-6]}$, 被广泛用
于石油化工热裂解 ${ }^{[7]}$ 、汽车尾气催化系统 ${ }^{[8-9]}$ 及热电 发动机 ${ }^{[10-11]}$ 等高温环境的催化剂或催化剂载体, 以 及航空航天领域的高温隔热材料 ${ }^{[12-13]}$ 。但是, 氧化 铝气凝胶强度较低, 难以制备成块体材料, 且制备

收稿日期: 2017-04-17; 收到修改稿日期：2017-06-04

基金项目: 国家重点基础研究发展计划(973, 2015CB057502); 国家自然科学基金(51673205)

National Basic Research Program of China (973 Program, 2015CB057502); National Natural Science Foundation of China (51673205)

作者简介: 杨景锋(1978-), 男, 博士. E-mail: jfyang@licp.cas.cn

通讯作者：王齐华, 研究员. E-mail: Wangqh@licp.cas.cn 
工艺复杂, 限制了其应用 ${ }^{[14-15]}$ 。对此, 研究人员开展 了许多针对性的研究。本文综述了近年来氧化铝气 凝胶的合成工艺, 及其在比表面积、热稳定性、隔 热性能及强度等性能改善方面取得的代表性研究成 果, 并对氧化铝气凝胶在高温催化及隔热领域的应 用前景进行了展望。

\section{1 氧化铝气凝胶的合成工艺}

\section{1 溶胶一凝胶工艺}

氧化铝气凝胶的合成需先将铝前驱体通过溶胶凝胶过程水解、缩聚形成醇凝胶, 再经过老化、溶 剂置换和干燥, 最后得到气凝胶。根据前驱体种类不 同, 醇凝胶一般通过有机铝醇盐法和无机铝盐法获得。

(1)有机铝醇盐中铝的反应活性较高, 需要采用 螯合剂来控制其凝胶过程 ${ }^{[16-17]}$ 。常用的螯合剂有乙 酰丙酮 ${ }^{[18]}$ 、乙酸 ${ }^{[19]}$ 、乙酰乙酸乙酯 $(E t a c)^{[20]}$ 等, 以 乙酰乙酸乙酯(Etac)为例, 其螯合机理如图 1 所示。 Etac 取代铝醇盐中的烷氧基后, 形成稳定的六元环 状络合物, 给水分子的亲核取代造成困难, 从而延 缓了铝醇盐的水解和缩聚速率 ${ }^{[21-22]}$ 。但是螯合剂的 加入也降低了凝胶的聚合程度, 制备的氧化铝气凝 胶强度较低, 容易碎裂成粉。

2013 年, $\mathrm{Zu}$ 等 ${ }^{[23]}$ 在以仲丁醇铝为前驱体制备醇 凝胶的过程中, 加入了丙酮和苯胺来控制水解及缩 聚反应的速率。丙酮和苯胺在酸性条件下会反应生 成水, 反应见(1) 式, 反应生成的副产物亚胺 $\left(\mathrm{C}_{6} \mathrm{H}_{5} \mathrm{~N}=\mathrm{C}\left(\mathrm{CH}_{3}\right)_{2}\right)$ 也被证明并不和铝醇盐发生反 应, 可以通过后期热处理除去。该研究使得有机铝 醇盐的凝胶过程更容易控制, 避免了螯合剂的加入 对凝胶强度的影响。

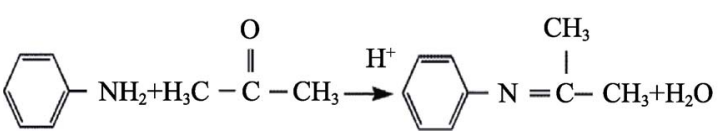

另外, 有机铝醇盐制备氧化铝气凝胶根据用水 量不同, 制备的气凝胶结构与性能有较大差异。当 水量过多, 制备的 $\mathrm{A}_{2} \mathrm{O}_{3}$ 气凝胶多为非晶结构, 凝 胶结构不稳定, 容易成粉 ${ }^{[24]}$; 当水量较少时, 制备 的 $\mathrm{A}_{2} \mathrm{O}_{3}$ 气凝胶为多晶勃姆石结构, 孔径比较均匀, 热稳定性好 ${ }^{[25-26]}$ 。

(2)无机铝盐价格低廉，一般制备的氧化铝气凝 胶颗粒及孔径较大, 热稳定性差 ${ }^{[27-28]} 。 2001$ 年, Gash 等 ${ }^{[29-30]}$ 发现以环氧丙烷作凝胶诱导剂容易控制凝胶 形成过程, 极大地推动了无机铝盐法合成 $\mathrm{A}_{2} \mathrm{O}_{3}$ 气 凝胶的研究。此后, 许多研究人员采用 $\mathrm{AlCl}_{3} \cdot 6 \mathrm{H}_{2} \mathrm{O}$

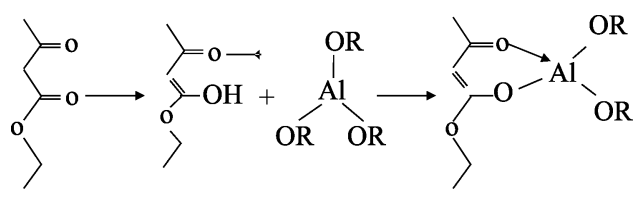

图 1 乙酰乙酸乙酯的螯合机理 ${ }^{[22]}$

Fig. 1 Ethyl acetoacetate chelating effect ${ }^{[22]}$

和 $\mathrm{Al}\left(\mathrm{NO}_{3}\right)_{3} \cdot 9 \mathrm{H}_{2} \mathrm{O}$ 作为前驱体, 环氧丙烷作为凝胶 剂, 制备了块体 $\mathrm{A}_{2} \mathrm{O}_{3}$ 气凝胶, 性能可以媲美有机 铝醇盐制备的 $\mathrm{A}_{2} \mathrm{O}_{3}$ 气凝胶 ${ }^{[17,31-33]}$ 。环氧丙烷在无 机铝盐的凝胶过程中涉及的反应如式(2 3)所示: 铝 离子先溶剂化形成水合铝离子, 进一步水解后提供 了质子(式 2)。环氧丙烷中氧原子的强亲核性使得其 与游离质子不断进行开环反应, 促进水合铝离子的 水解和聚合(式 3), 同时反应体系中的阴离子 $\mathrm{X}^{-}$(如 $\mathrm{Cl}^{-}$或 $\mathrm{NO}_{3}^{-}$) 也参与开环反应消耗氧离子。

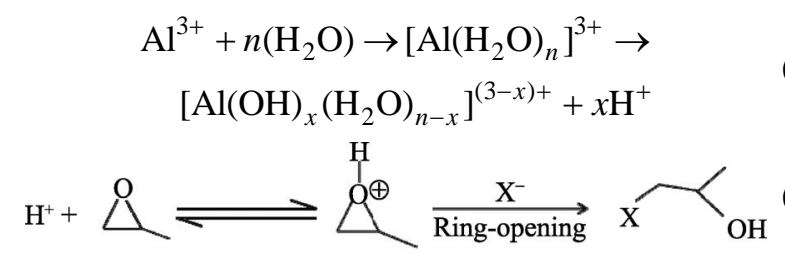

\section{2 氧化铝醇凝胶的干燥工艺}

氧化铝醇凝胶的干燥一般有超临界干燥、常压 干燥及冷冻干燥等方式, 表 1 为不同干燥工艺的工 艺特点及其制备的气凝胶性能, 从中可以看出干燥 工艺对气凝胶性能有重要影响, 因此研究人员在改 进干燥工艺以及发展新的干燥工艺方面做出了不解 努力。2010 年, Michael 等 ${ }^{[6]}$ 将包含湿凝胶的不锈钢 模具用石墨片和 Capton 薄膜密封, 用液压机对模具 加压并加热，使得模具内的溶剂达到超临界条件, 然后通过一定的泄压速率实现介质溶剂的快速超临 界干燥(RSCE)(图 2)。该方法可一次干燥多个气凝 胶产品，提高了氧化铝气凝胶的制备效率,将以往在 高压釜上进行的超临界干燥转移到液压机和模具上 来，降低了操作风险，也节省了时间。

表 1 几种干燥方式的特点

Table 1 Characteristics of several drying methods

\begin{tabular}{|c|c|c|}
\hline \multirow{2}{*}{$\begin{array}{l}\text { Drying } \\
\text { method }\end{array}$} & \multicolumn{2}{|c|}{ Characteristics } \\
\hline & Drying process & Alumina aerogel \\
\hline $\begin{array}{l}\text { Supercritical } \\
\text { drying }\end{array}$ & $\begin{array}{l}\text { Short drying period, } \\
\text { high synthesis cost }\end{array}$ & $\begin{array}{l}\text { Low density, high porosity } \\
\text { and specific surface area, low } \\
\text { volume shrinkage }^{[34-35]}\end{array}$ \\
\hline $\begin{array}{l}\text { Ambient } \\
\text { pressure } \\
\text { drying }\end{array}$ & $\begin{array}{l}\text { Long drying period, } \\
\text { inexpensive synthe- } \\
\text { sis cost, complicated } \\
\text { drying process }\end{array}$ & $\begin{array}{l}\text { High volume shrinkage, large } \\
\text { pore size, high density and } \\
\text { low specific surface area }\end{array}$ \\
\hline $\begin{array}{l}\text { Freeze } \\
\text { drying }\end{array}$ & $\begin{array}{l}\text { Long drying period, } \\
\text { inexpensive synthe- } \\
\text { sis cost }\end{array}$ & $\begin{array}{l}\text { High volume shrinkage, large } \\
\text { pore size, high density and } \\
\text { low specific surface area }\end{array}$ \\
\hline
\end{tabular}




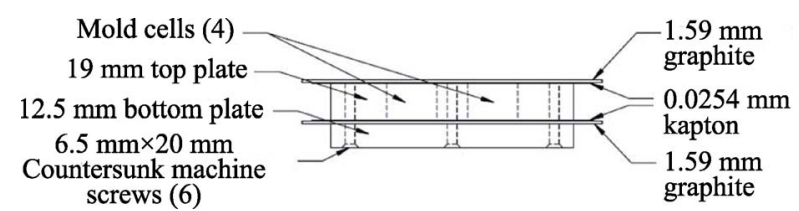

图 2 快速超临界干燥的模具装配示意图 ${ }^{[6]}$

Fig. 2 Schematic diagram of the mold assembly used for $\operatorname{RSCE}^{[6]}$

超临界干燥过程中溶剂先从液体转变成气体, 所产生的表面张力会影响凝胶网络中的孔结构。 2016 年, Yang 等 ${ }^{[38]}$ 通过控制乙醇超临界干燥过程的 温度与压力, 使得乙醇从液体直接到达超临界状态, 越过气相区域, 消除了液一气转变过程中表面张力 对孔的影响, 从而获得高比表面积和热稳定性的氧 化铝气凝胶(图 3)。该方法也可用于二氧化碳等其他 介质溶剂的超临界干燥, 可以进一步缩短气凝胶的 合成时间，并提高其性能

2014 年, Ren 等 ${ }^{[39]}$ 发展了一种有机溶剂升华干 燥(OSSD)方法。该方法采用低表面张力、高凝固点 且容易升华的有机溶剂, 如乙腈、叔丁醇等, 代替醇 凝胶结构中的溶剂和水, 然后在低真空下干燥。干 燥期间醇凝胶表面的溶剂首先蒸发, 蒸发吸收热量 导致周围溶剂从液体转变成固体, 然后再通过升华

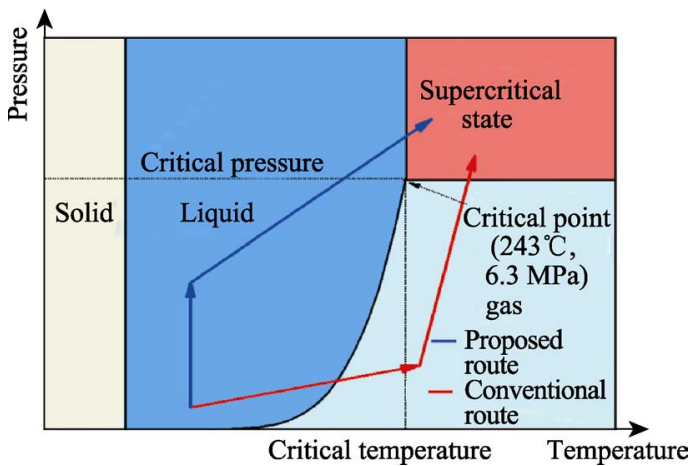

图 3 乙醇的相图 ${ }^{[35]}$

Fig. 3 Phase diagram of ethanol ${ }^{[35]}$
将溶剂除掉(图 4)。升华干燥避免了气一液界面的出 现，消除了表面张力对孔的影响。采用该方法能够 制备出完整的块体氧化铝气凝胶, 有较高强度, 但 体积收缩仍较大。

常压干燥工艺复杂, 干燥周期较长 ${ }^{[35-37]}$ 。 Xu 等 ${ }^{[31]}$ 在溶胶一凝胶过程中通过添加干燥化学控制剂 与结构支撑剂, 采用环氧丙烷为凝胶诱导剂, 经老 化及溶剂交换, 实现了常压干燥, 制备的氧化铝气 凝胶与超临界干燥工艺得到的氧化铝气凝胶性能相 近, 制备周期长达 $8 \mathrm{~d}$ 。考虑到氧化铝气凝胶的连续 化及规模化生产, 常压干燥仍然是首选方式, 但仍 需要进一步研究并简化。

\section{2 氧化铝气凝胶的性能}

\section{$2.1 \mathrm{Al}_{2} \mathrm{O}_{3}$ 气凝胶的比表面积和热稳定性}

$\mathrm{Al}_{2} \mathrm{O}_{3}$ 气凝胶虽然具有大的比表面积及热稳定 性, 但离实际应用还有一定差距。 $\mathrm{Al}_{2} \mathrm{O}_{3}$ 气凝胶在 $1000^{\circ} \mathrm{C}$ 以上的 $\gamma$ 相到 $\alpha$ 相的转变伴随着较大的比表 面积损失和体积收缩，限制了其应用 ${ }^{[4]}$ 。研究人员通 过向 $\mathrm{A}_{2} \mathrm{O}_{3}$ 气凝胶中掺杂 $\mathrm{La}, \mathrm{Y}, \mathrm{Ba}, \mathrm{Si}$ 等多种元素, 使其比表面积和热稳定性均有不同程度的改善 ${ }^{[40-44]}$ 。 Alphonse 等 ${ }^{[45]}$ 对 $\mathrm{A} 1_{2} \mathrm{O}_{3}$ 气凝胶掺杂 $\mathrm{La}$ 后, 发现 $1000^{\circ} \mathrm{C}$ 时其比表面积为 $180 \mathrm{~m}^{2} / \mathrm{g} ; 1200^{\circ} \mathrm{C}$ 时其比表 面积为 $70 \mathrm{~m}^{2} / \mathrm{g}$, 热稳定性得到了显著改善, 这是由 于形成的 $\mathrm{La}-\mathrm{Al}$ 混合氧化物 $\left(\mathrm{LaAl}_{11} \mathrm{O}_{18}\right.$ 或 $\left.\mathrm{LaAlO}_{3}\right)$ 抑 制了氧化铝的烧结。 $\mathrm{Wu}$ 等 ${ }^{[46]}$ 研究显示 $\mathrm{Si}$ 掺杂的 $\mathrm{A}_{2} \mathrm{O}_{3}$ 气凝胶的比表面积 $1000{ }^{\circ} \mathrm{C}$ 时为 $373 \mathrm{~m}^{2} / \mathrm{g}$, $1200{ }^{\circ} \mathrm{C}$ 时为 $120 \mathrm{~m}^{2} / \mathrm{g}$; 即使在 $1400{ }^{\circ} \mathrm{C}$ 时仍保持 $40 \mathrm{~m}^{2} / \mathrm{g}^{[41]}$, 因此 $\mathrm{Si}$ 的掺杂效果比 La 更为显著。分 析认为, $\mathrm{Si}$ 对 $\mathrm{A}_{2} \mathrm{O}_{3}$ 气凝胶热稳定性的改善主要是 由于高温下 $\mathrm{SiO}_{2}$ 和 $\mathrm{A}_{1} \mathrm{O}_{3}$ 会形成莫来石结构，抑制 $\mathrm{A}_{2} \mathrm{O}_{3}$ 的 $\mathrm{a}$ 相变, 从而保持大的比表面积 ${ }^{[38,41-42,47]}$ 。

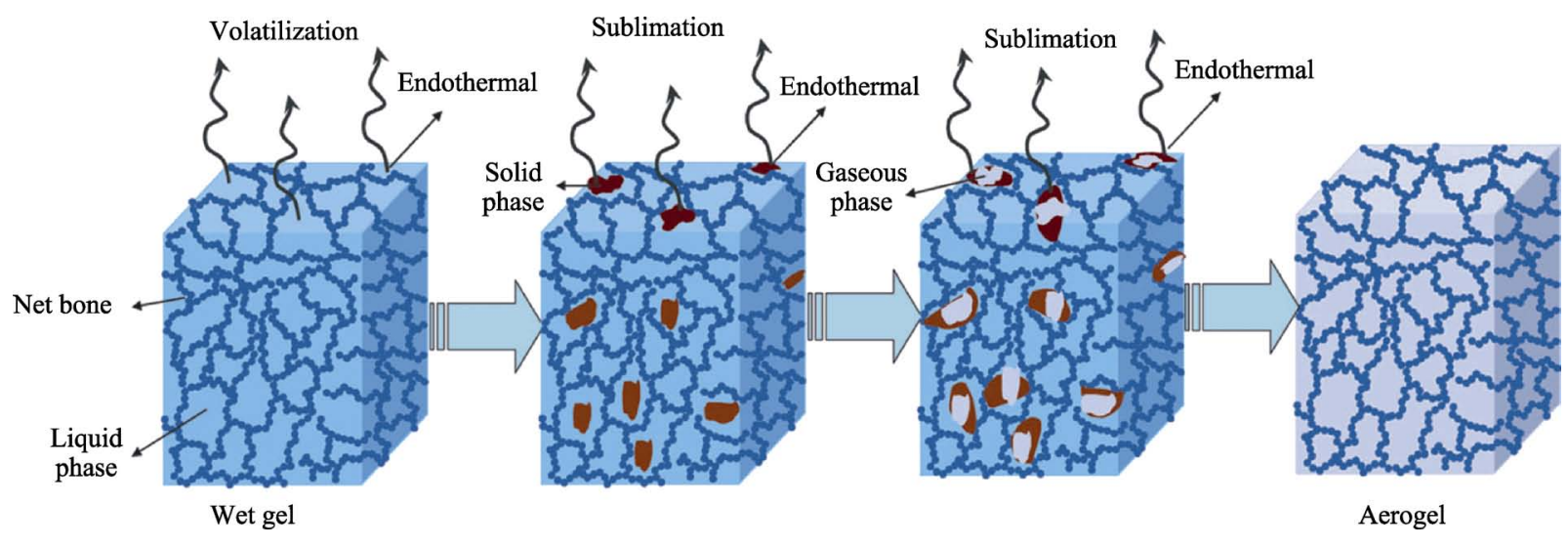

图 4 OSSD 制备气凝胶的干燥机理示意图 ${ }^{[39]}$

Fig. 4 Mechanism illustration of the formation of aerogels by OSSD $^{[39]}$ 


\section{$2.2 \mathrm{Al}_{2} \mathrm{O}_{3}$ 气凝胶的强度及隔热性能}

纯 $\mathrm{Al}_{2} \mathrm{O}_{3}$ 气凝胶强度较低, 难以形成块体材料, 影响其使用。通过结构设计、改性等方法来提高强 度一直是 $\mathrm{Al}_{2} \mathrm{O}_{3}$ 气凝胶研究的一个重要内容。2014 年, $\mathrm{Zu}$ 等 ${ }^{[48]}$ 以仲丁醇铝为前驱体制备的醇凝胶, 经 化学液相沉积 $\mathrm{Al}_{2} \mathrm{O}_{3}-\mathrm{SiO}_{2}$ 和 $\mathrm{SiO}_{2}$ 溶胶后, 超临界干 燥得到了核壳结构 $\mathrm{Al}_{2} \mathrm{O}_{3}$ 气凝胶。他们发现核壳结 构的 $\mathrm{Al}_{2} \mathrm{O}_{3}$ 气凝胶压缩模量相比纯 $\mathrm{Al}_{2} \mathrm{O}_{3}$ 气凝胶 (1.7 MPa)提高了几乎 4 倍, 达到 $6.7 \mathrm{MPa}$ (图 5(a)); $1300^{\circ} \mathrm{C}$ 热处理 $2 \mathrm{~h}$ 后, 形貌变化不大, 线收缩仅 $4 \%$, 显示了优异的热稳定性(图 5(b)), 常温下导热系数 $0.042 \mathrm{~W} /(\mathrm{m} \cdot \mathrm{K})$, 高温隔热性能优于莫来石纤维隔热 材料。研究认为, 核壳结构增强了气凝胶纳米粒子 的强度, 抑制了高温热处理过程中晶粒的生长和相 变, 这为提高 $\mathrm{Al}_{2} \mathrm{O}_{3}$ 气凝胶材料的热稳定性和机械 强度提供了新的思路和方法。

$\mathrm{CaO}$ 等 ${ }^{[49]}$ 用 $\mathrm{AlCl}_{3} \cdot 6 \mathrm{H}_{2} \mathrm{O}$ 为前驱体, 引入纳米凹 凸棒石 (Attapulgite, ATP), 环氧丙烷为凝胶诱导剂, 常压干燥制备了 $\mathrm{ATP} / \mathrm{Al}_{2} \mathrm{O}_{3}$ 复合气凝胶。研究发现, $\mathrm{ATP} / \mathrm{Al}_{2} \mathrm{O}_{3}$ 复合气凝胶压缩强度随 ATP 含量增加先 增加后降低, ATP 含量为 $26 \%$ 时, 压缩强度最高达
到 $75.44 \mathrm{MPa}$ 。研究认为, $\mathrm{A}_{2} \mathrm{O}_{3}$ 纳米粒子能粘附在 ATP 的棒状结构单元上, 然后随机组装形成 $\mathrm{ATP} / \mathrm{Al}_{2} \mathrm{O}_{3}$ 复合气凝胶的网络骨架, 大幅提高了 $\mathrm{A}_{2} \mathrm{O}_{3}$ 气凝胶的强度。该研究对于 $\mathrm{A}_{2} \mathrm{O}_{3}$ 气凝胶的 合成和应用具有重要意义, 但是强度的大幅增高, 使得其结构更加致密，比表面积和隔热性能降低， 因此, $\mathrm{ATP} / \mathrm{Al}_{2} \mathrm{O}_{3}$ 复合气凝胶的强度和比表面积、导 热系数以及热稳定性的制约关系还需进一步研究。

$\mathrm{Wu}$ 等 ${ }^{[50]}$ 还采用 $\mathrm{AlCl}_{3} \cdot 6 \mathrm{H}_{2} \mathrm{O}$ 作为铝源, 间苯二 酚 $(\mathrm{R})$ 和甲醛 $(\mathrm{F})$ 作为碳源, $\mathrm{CO}_{2}$ 超临界干燥制备了 $\mathrm{RF} / \mathrm{Al}_{2} \mathrm{O}_{3}$ 气凝胶, 然后在氩气保护下 $800 ~ 1500^{\circ} \mathrm{C}$ 进 行了热处理, 将 $\mathrm{RF} / \mathrm{Al}_{2} \mathrm{O}_{3}$ 气凝胶转变成 $\mathrm{C} / \mathrm{Al}_{2} \mathrm{O}_{3}$ 气 凝胶。结果发现, 先制备的 $\mathrm{RF} / \mathrm{Al}_{2} \mathrm{O}_{3}$ 中由于存在 $-\mathrm{CH}_{2}$ 或者 $-\mathrm{CH}_{2} \mathrm{OCH}_{2}$ 柔性单元, 压缩模量较低, 而 在碳化后转变为 $\mathrm{C} / \mathrm{Al}_{2} \mathrm{O}_{3}$ 后, 强度大幅提高, 压缩 模量可以达到 $90 \mathrm{MPa}$ (图 6(a)), 并指出 $\mathrm{C}_{1} \mathrm{Al}_{2} \mathrm{O}_{3}$ 气凝 胶强度的大幅提高主要因为热处理提高了 $\mathrm{Al}_{2} \mathrm{O}_{3}$ 气 凝胶的网络骨架强度, 同时无定型 $\mathrm{C}$ 覆盖在 $\mathrm{Al}_{2} \mathrm{O}_{3}$ 纳米粒子上, 阻碍了 $\mathrm{Al}_{2} \mathrm{O}_{3}$ 纳米粒子的烧结, $\alpha-\mathrm{Al}_{2} \mathrm{O}_{3}$ 的 相变被延缓到 $1500^{\circ} \mathrm{C}$ 以上。在室温到 $1500^{\circ} \mathrm{C}$ 范围, $\mathrm{C} / \mathrm{Al}_{2} \mathrm{O}_{3}$ 气凝胶的导热系数为 $0.033 \sim 0.049(\mathrm{~W} /(\mathrm{m} \cdot \mathrm{K})$ ),
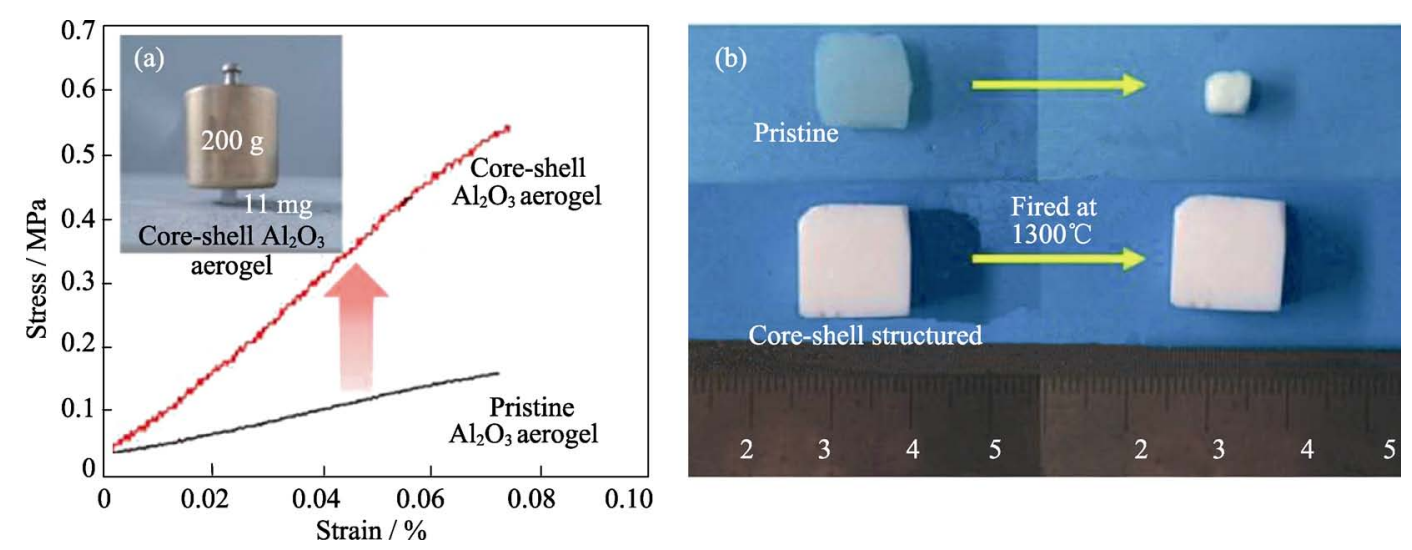

图 $5 \mathrm{Al}_{2} \mathrm{O}_{3}$ 气凝胶的应力应变曲线( $\mathrm{a}$ 和高温热处理前后 $\mathrm{Al}_{2} \mathrm{O}_{3}$ 气凝胶(b) ${ }^{[48]}$

Fig. 5 Stress-strain curves of $\mathrm{Al}_{2} \mathrm{O}_{3}$ aerogels (a) and $\mathrm{Al}_{2} \mathrm{O}_{3}$ aerogels before and after heat treatment at high temperature (b) ${ }^{[48]}$
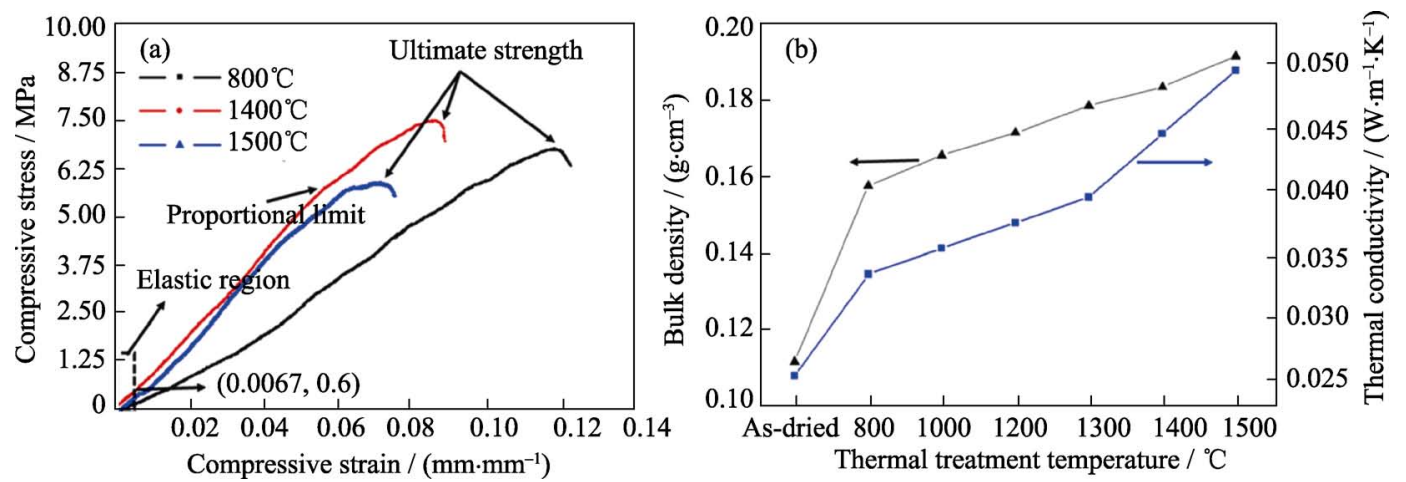

图 $6 \mathrm{C} / \mathrm{Al}_{2} \mathrm{O}_{3}$ 气凝胶不同温度热处理后的应力-应变曲线 $(\mathrm{a})$ 和密度、导热系数变化(b) ${ }^{[50]}$

Fig. 6 Compressive stress-strain curves (a) and bulk densities and thermal conductivities (b) of $\mathrm{C} / \mathrm{Al}_{2} \mathrm{O}_{3}$ aerogels heat treated at different temperatures ${ }^{[50]}$ 
密度约 $0.1 \sim 0.2 \mathrm{~g} / \mathrm{cm}^{3}$ (图 6(b)), 显示出优异的强度、 热稳定性以及隔热性能, 为惰性气氛条件下隔热材 料的应用提供了参考。

\section{3 纤维 $/ \mathrm{Al}_{2} \mathrm{O}_{3}$ 气凝胶复合材料}

2015年, He 等 ${ }^{[51]}$ 在仲丁醇铝为前驱体制备的溶 胶中, 引入了纤维素(Cellulose), 经冷冻干燥制备了 $\mathrm{Al}_{2} \mathrm{O}_{3}$-Cellulose 气凝胶材料(ACA), 研究发现 ACA 热稳定性与 $\mathrm{Al}_{2} \mathrm{O}_{3}$ 气凝胶比较接近, 在 $1200^{\circ} \mathrm{C}$ 热处 理后体积收缩约 15\%, ACA 能保持完整块状。引入 Cellulose 改善了 $\mathrm{Al}_{2} \mathrm{O}_{3}$ 气凝胶的强度, 在纤维素含 量为 $2 \%$ 时压缩强度为 $0.553 \mathrm{MPa}$ (图 7), 并在受压形 变 $20 \%$ 以下具有部分形状恢复性能, 展示出部分柔 性(图 7 插图), 克服了长久以来 $\mathrm{Al}_{2} \mathrm{O}_{3}$ 气凝胶的易碎 易掉渣的缺点, 为 $\mathrm{Al}_{2} \mathrm{O}_{3}$ 气凝胶的应用提供了新方 法, 但合成周期过长, 仍需进一步研究。

$\mathrm{Xu}$ 等 ${ }^{[52]}$ 考察了莫来石纤维 $/ \mathrm{Al}_{2} \mathrm{O}_{3}-\mathrm{SiO}_{2}$ 气凝胶 材料的高温隔热性能。由于高温下热辐射对热传导 影响较大, 一般在气凝胶中添加红外遮蔽剂来减小 热辐射的措施。Xu等在莫来石纤维表面涂覆了 $\mathrm{SiC}$ 涂层,再制备莫来石纤维 $/ \mathrm{Al}_{2} \mathrm{O}_{3}-\mathrm{SiO}_{2}$ 气凝胶材料, 解 决了 $\mathrm{SiC}$ 作为红外遮蔽剂在纤维/气凝胶复合材料中 的分散问题。结果发现, 前驱物浓度为 $10 \%$ 时, 具 有 $\mathrm{SiC}$ 涂层的纤维/气凝胶复合材料的有效比消光系 数达到 $56.3 \mathrm{~m}^{2} / \mathrm{kg}$ (图 8), 使得其在 $1000^{\circ} \mathrm{C}$ 的导热系 数为 $0.049 \mathrm{~W} /(\mathrm{m} \cdot \mathrm{K})$, 表明 $\mathrm{SiC}$ 涂层可以有效遮蔽高 温热辐射, 制备的复合材料具有优异的隔热性能, 为氧化铝气凝胶在高温隔热领域的应用提供了 可能。

\section{3 氧化铝气凝胶的应用}

氧化铝气凝胶优异的热稳定性和大的比表面积,

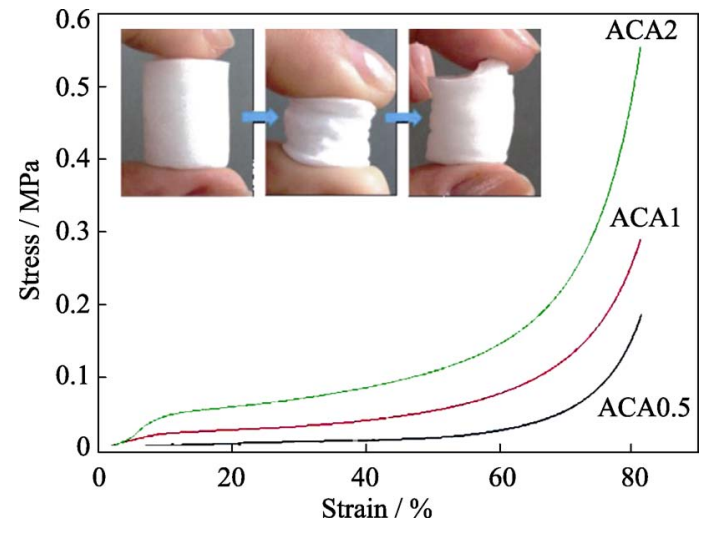

图 7 不同纤维素含量 ACA 的应力应变曲线(ACA0.5, ACA1, ACA2 分别为纤维素含量 $0.5 \%, 1 \%, 2 \%)^{[51]}$

Fig. 7 Stress-strain curves of ACAs with different contents of cellulose (ACA0.5, ACA1 and ACA2 corresponding to the cellulose content of $0.5 \mathrm{wt} \%, 1 \mathrm{wt} \%$ and $2 \mathrm{wt} \%$, respectively $)^{[51]}$

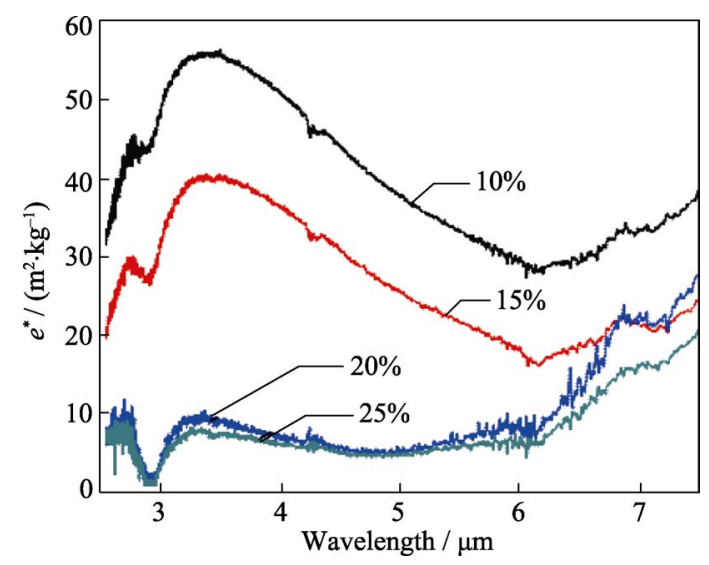

图 8 不同前驱物浓度得到的 $\mathrm{SiC}$ 涂层的莫来石纤维 $/ \mathrm{Al}_{2} \mathrm{O}_{3}-$ $\mathrm{SiO}_{2}$ 气凝胶的有效比消光系数 ${ }^{[52]}$

Fig. 8 Effective specific extinction coefficient of $\mathrm{Al}_{2} \mathrm{O}_{3}-\mathrm{SiO}_{2}$ aerogel composites reinforced by SiC-coated mullite fibers prepared with different concentration of precursor $^{[52]}$

使其在催化剂及催化剂载体方面有广泛应用。Yoo 等 ${ }^{[33]}$ 研究了 $\mathrm{Ni}^{-} \mathrm{Al}_{2} \mathrm{O}_{3}$ 气凝胶催化剂在天然气蒸汽 重整制氢过程中的催化性能, 发现 $\mathrm{Ni}$ 在 $\mathrm{Al}_{2} \mathrm{O}_{3}$ 气凝 胶中可以高度分散并具有较小的粒径, 使得催化性 能得到显著提升。此外, $\mathrm{Ni}-\mathrm{Al}_{2} \mathrm{O}_{3}$ 气凝胶在 $\mathrm{CH}_{4}-\mathrm{CO}_{2}$ 重整过程中能抑制表面积碳导致的催化剂失活, 具 有好的催化活性和稳定性 ${ }^{[54-55]}$ 。Ozawa 等 ${ }^{[8]}$ 研究显 示 $\mathrm{La} / \mathrm{CuO}_{x}-\mathrm{Al}_{2} \mathrm{O}_{3}$ 气凝胶用作汽车尾气催化剂, 在 $1000^{\circ} \mathrm{C}$ 高温下, $\mathrm{NO}$ 和碳氢化合物的去除效率得到 大幅提升。Mi 等 ${ }^{[56]}$ 在合成单壁碳纳米管中发现, $\mathrm{Fe} / \mathrm{Mo} / \mathrm{A}_{2} \mathrm{O}_{3}$ 气凝胶 $600^{\circ} \mathrm{C}$ 敾烧后催化活性最高。

作为隔热材料, 氧化铝气凝胶可用于航空航天 领域, 如美国 NASA 研究双组分的 $\mathrm{Al}_{2} \mathrm{O}_{3}-\mathrm{SiO}_{2}$ 气凝 胶用于航天飞行器的热防护系统 ${ }^{[2,13]}$; Gao 等 ${ }^{[57]}$ 研 究显示莫来石纤维复合氧化铝气凝胶隔热材料在高 温 $1000^{\circ} \mathrm{C}$ 时导热系数比莫来石纤维下降了 $47.5 \%$, 比氧化硅气凝胶下降了 $13.4 \%$, 可将其用于航天飞 行器的热防护系统、导弹舵机舱隔热和热电池隔热

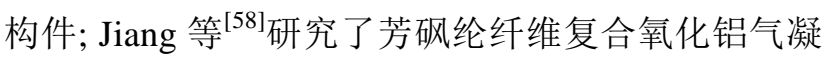
胶用作隔热耐火消防服, 用垂直燃烧法测试后发现 复合材料损毁长度大大降低, 具有优异的阻燃性能 和隔热性能; Xie 等 ${ }^{[59]}$ 研究了聚氨酯泡沫中引入氧 化铝气凝胶, 得到的复合材料的阻燃和隔热性能得 到显著提高, 可用于建筑、电力工业等领域的阻燃 隔热材料。

\section{4 结束语}

尽管近年来人们对氧化铝气凝胶的合成工艺以 及性能改善的研究已取得了很大的进展, 但随着科 技的发展, 氧化铝气凝胶离实际高温隔热及催化剂 
应用仍存在一定差距。作为催化剂或催化剂载体, 氧化铝气凝胶需要通过掺杂改性、与高催化活性的 组分结合来进一步提高比表面积、热稳定性以及催 化活性。在高温隔热领域, 纤维增强氧化铝气凝胶 仍是未来高温隔热材料的发展方向, 其高强度和低 导热系数、低密度之间的制约关系需要通过进一步 研究来突破, 高温下的热辐射及热传导对实际隔热 性能的影响还需深入研究, 从而发展出集高强度、 低导热系数、低密度为一体的耐高温纤维/氧化铝气 凝胶复合材料, 解决高温环境的隔热难题。此外, 研 究氧化铝气凝胶现有的合成工艺, 特别是简化干燥 工艺, 制备出较大尺寸的纤维/氧化铝气凝胶材料, 是实现氧化铝气凝胶产业化的关键。

\section{参考文献:}

[1] AMEEN K B, RAJASEKAR K, RAJASEKHARAN T, et al. The effect of heat-treatment on the physico-chemical properties of silica aerogel prepared by sub-critical drying technique. Journal of Sol-Gel Science and Technology, 2008, 45(1): 9-15.

[2] HURWITZ F I, GUO H, ROGERS R B, et al. Influence of Ti addition on boehmite-derived aluminum silicate aerogels: structure and properties. Journal of Sol-Gel Science and Technology, 2012, 64(2): 367-374.

[3] JUHL S J, DUNN N J H, CARROLL M K, et al. Epoxide-assisted alumina aerogels by rapid supercritical extraction. Journal of Non-Crystalline Solids, 2015, 426: 141-149.

[4] SMITH S J, HUANG B, BARTHOLOMEW C H, et al. La-dopant location in La-doped $\gamma-\mathrm{Al}_{2} \mathrm{O}_{3}$ nanoparticles synthesized using a novel one-pot process. The Journal of Physical Chemistry C, 2015, 119(44): 25053-25062.

[5] WEI Q, CHEN Z X, WANG Z H, et al. Effect of La, Ce, Y and B addition on thermal stability of unsupported alumina membranes. Journal of alloys and compounds, 2005, 387(1): 292-296.

[6] JR. M S B, ANDERSON A M, CARROLL M K. Alumina aerogels prepared via rapid supercritical extraction. J. Sol-Gel Sci. Technol., 2010, 53(2010): 216-226.

[7] AL-YASSIR N, LE VAN MAO R. Thermal stability of alumina aerogel doped with yttrium oxide, used as a catalyst support for the thermocatalytic cracking (TCC) process: an investigation of its textural and structural properties. Applied Catalysis A: General, 2007, 317(2): 275-283.

[8] OZAWA M. Thermal stabilization of catalytic compositions for automobile exhaust treatment through rare earth modification of alumina nanoparticle support. Journal of Alloys and Compounds, 2006, 408-412: 1090-1095.

[9] CHEN X, LIU Y, NIU G, et al. High temperature thermal stabilization of alumina modified by lanthanum species. Applied Catalysis A: General, 2001, 205(1): 159-172.

[10] GUIDOTTI R A, REED S T, ASHLEY C S, et al. Evaluation of Aerogel Materials for High-Temperature Batteries. SAE International, 1999.

[11] AEgerter M A, LEVEntis N, KOEBEL M M. Aerogels handbook. Springer Science \& Business Media, 2011.

[12] JONES S M. Aerogel: Space exploration applications. Journal of Sol-Gel Science and Technology, 2006, 40(2/3): 351-357.

[13] HURWITZ F I. Thermal Protection Systems (TPSs). In book:
Richard Blockley, Wei Shyy, Encyclopedia of Aerospace Engineering. John Wiley \& Sons, Ltd. 2010: 1-13.

[14] YANG X, WEI J, SHI D, et al. Comparative investigation of creep behavior of ceramic fiber-reinforced alumina and silica aerogel. Materials Science and Engineering: A, 2014, 609: 125-130.

[15] YANG J, LI S, LUO Y, et al. Compressive properties and fracture behavior of ceramic fiber-reinforced carbon aerogel under quasi-static and dynamic loading. Carbon, 2011, 49(5): 1542-1549.

[16] ZU G, SHEN J, WEI X, et al. Preparation and characterization of monolithic alumina aerogels. Journal of Non-Crystalline Solids, 2011, 357(15): 2903-2906.

[17] BAUMANN T F, GASH A E, CHINN S C, et al. Synthesis of high-surface-area alumina aerogels without the use of alkoxide precursors. Chemistry of Materials, 2005, 17(2): 395-401.

[18] JI L, LIN J, TAN K, et al. Synthesis of high-surface-area alumina using aluminum tri-sec-butoxide-2, 4-pentanedione-2-propanolnitric acid precursors. Chemistry of Materials, 2000, 12(4): 931-939.

[19] POCO J, SATCHER J, HRUBESH L. Synthesis of high porosity, monolithic alumina aerogels. Journal of Non-Crystalline Solids, 2001, 285(1): 57-63.

[20] PIERRE A, BEGAG R, PAJONK G. Structure and texture of alumina aerogel monoliths made by complexation with ethyl acetoacetate. Journal of Materials Science, 1999, 34(20): 4937-4944.

[21] PIERRE A C, PAJONK G M. Chemistry of aerogels and their applications. Chemical Reviews, 2002, 102(11): 4243-4266.

[22] GAO Q F, ZHANG C R, FENG J, et al. Preparation of low density, monolithic alumina aerogels. Chinese Journal of Inorganic Chemistry, 2008, 9: 19-25.

[23] ZU G, SHEN J, ZOU L, et al. Nanoengineering super heat-resistant, strong alumina aerogels. Chemistry of Materials, 2013, 25(23): 4757-4764.

[24] JANOSOVITS U, ZIEGLER G, SCHARF U, et al. Structural characterization of intermediate species during synthesis of $\mathrm{Al}_{2} \mathrm{O}_{3}$ aerogels. Journal of Non-crystalline Solids, 1997, 210(1): 1-13.

[25] HORIUCHI T, CHEN L, OSAKI T, et al. A novel alumina catalyst support with high thermal stability derived from silica-modified alumina aerogel. Catalysis Letters, 1999, 58(2/3): 89-92.

[26] OSAKI T, NAGASHIMA K, WATARI K, et al. Silica-doped alumina cryogels with high thermal stability. Journal of Non-crystalline Solids, 2007, 353(24): 2436-2442.

[27] HE FEI, HE XIAO-DONG, LI YAO. $\mathrm{Al}_{2} \mathrm{O}_{3}$ Xerogels synthesized by inorganic salt and organic alkoxide. Journal of the Chinese Ceramic Society, 2006, 34(9): 1093-1097.

[28] HAO Z, LIU H, GUO B, et al. Sol-Gel synthesis of alumina using inorganic salt precursor. Acta Physico-Chimica Sinica, 2007, 23(3): 289-294.

[29] GASH A E, TILLOTSON T M, SATCHER J H, et al. Use of epoxides in the Sol-Gel synthesis of porous iron (III) oxide monoliths from Fe (III) salts. Chemistry of Materials, 2001, 13(3): 999-1007.

[30] GASH A E, TILLOTSON T M, SATCHER JR J H, et al. New Sol-Gel synthetic route to transition and main-group metal oxide aerogels using inorganic salt precursors. Journal of Non-Crystalline Solids, 2001, 285(1): 22-28.

[31] XU ZI-JIE, GAN LI-HUA, PANG YING-CONG, et al. Preparation of $\mathrm{Al}_{2} \mathrm{O}_{3}$ bulk aerogels by non-supercritical fluid drying technology. Acta Phys.-Chim.Sin., 2005, 21(2): 221-224.

[32] WU X, SHAO G, SHEN X, et al. Novel $\mathrm{Al}_{2} \mathrm{O}_{3}-\mathrm{SiO}_{2}$ composite aerogels with high specific surface area at elevated temperatures with different alumina/silica molar ratios prepared by a non-alkoxide Sol-Gel method. RSC Adv, 2016, 6(7): 5611-5620.

[33] JUHL S J, DUNN N J H, CARROLL M K, et al. Epoxide-assisted alumina aerogels by rapid supercritical extraction. Journal of 
Non-Crystalline Solids, 2015, 426: 141-149.

[34] SHEWALE P M, RAO A V, RAO A P. Effect of different trimethyl silylating agents on the hydrophobic and physical properties of silica aerogels. Applied Surface Science, 2008, 254(21): 6902-6907.

[35] HILONGA A, KIM J K, SARAWADE P B, et al. Low-density TEOS-based silica aerogels prepared at ambient pressure using isopropanol as the preparative solvent. Journal of Alloys and Compounds, 2009, 487(1): 744-750.

[36] WU L, HUANG Y, WANG Z, et al. Fabrication of hydrophobic alumina aerogel monoliths by surface modification and ambient pressure drying. Applied Surface Science, 2010, 256(20): 59735977.

[37] TALEBI MAZRAEH-SHAHI Z, MOUSAVI SHOUSHTARI A, BAHRAMIAN A R, et al. Synthesis, pore structure and properties of polyurethane/silica hybrid aerogels dried at ambient pressure. Journal of Industrial and Engineering Chemistry, 2015, 21: 797-804.

[38] YANG J, WANG Q, WANG T, et al. Facile one-step precursorto-aerogel synthesis of silica-doped alumina aerogels with high specific surface area at elevated temperatures. Journal of Porous Materials, 2017, 24(4): 889-897.

[39] REN L, CUI S, CAO F, et al. An easy way to prepare monolithic inorganic oxide aerogels. Angew Chem. Int. Ed. Engl., 2014, 53(38): 10147-10149.

[40] ZHONG X, ZHAO H, LIU C, et al. Improvement in thermal shock resistance of gadolinium zirconate coating by addition of nanostructured yttria partially-stabilized zirconia. Ceramics International, 2015, 41(6): 7318-7324.

[41] TATSURO H, TOSHIHIKO O, TOYOHIKO S, et al. Maintenance of large surface area of alumina aerogel heated at elevated temperature above 1300 by preparing silica-containing aerogel. Journal of Non-Crystalline Solids, 2001, 291(3): 187-198.

[42] MIZUSHIMA Y, HORI M. Preparation of heat-resistant alumina aerogels. Journal of Materials Research, 1993, 8(11): 2993-2999.

[43] YANG J, WANG Q, WANG T, et al. Rapid preparation process, structure and thermal stability of lanthanum doped alumina aerogels with a high specific surface area. RSC $A d v, 2016,6$ (31): 26271-26279.

[44] BANG Y, SEO J G, SONG I K. Hydrogen production by steam reforming of liquefied natural gas (LNG) over mesoporous $\mathrm{Ni}-$ $\mathrm{La}-\mathrm{Al}_{2} \mathrm{O}_{3}$ aerogel catalysts: effect of La content. International Journal of Hydrogen Energy, 2011, 36(14): 8307-8315.

[45] ALPHONSE P, FAURE B. Thermal stabilization of alumina modified by lanthanum. Microporous and Mesoporous Materials, 2014, 196: 191-198.

[46] WU X, SHAO G, CUI S, et al. Synthesis of a novel $\mathrm{Al}_{2} \mathrm{O}_{3}-\mathrm{SiO}_{2}$ composite aerogel with high specific surface area at elevated temperatures using inexpensive inorganic salt of aluminum. Ceramics International, 2016, 42(1): 874-882.
[47] WU X, SHAO G, SHEN X, et al. Novel $\mathrm{A}_{12} \mathrm{O}_{3}-\mathrm{SiO}_{2}$ composite aerogels with high specific surface area at elevated temperatures with different alumina/silica molar ratios prepared by a non-alkoxide Sol-Gel method. RSC Adv., 2016, 6(7): 5611-5620.

[48] ZU G, SHEN J, WANG W, et al. Robust, highly thermally stable, core-shell nanostructured metal oxide aerogels as high-temperature thermal superinsulators, adsorbents, and catalysts. Chemistry of Materials, 2014, 26(19): 5761-5772.

[49] CAO F, REN L, LI X. Synthesis of high strength monolithic alumina aerogels at ambient pressure. RSC Adv., 2015, 5(23): 1802518028.

[50] WU X, ZHONG Y, KONG Y, et al. Preparation and characterization of $\mathrm{C} / \mathrm{Al}_{2} \mathrm{O}_{3}$ composite aerogel with high compressive strength and low thermal conductivity. Journal of Porous Materials, 2015, 22(5): 1235-1243.

[51] HE F, SUI C, HE X, et al. Facile synthesis of strong aluminacellulose aerogels by a freeze-drying method. Materials Letters, 2015, 152: 9-12.

[52] XU L, JIANG Y, FENG J, et al. Infrared-opacified $\mathrm{Al}_{2} \mathrm{O}_{3}-\mathrm{SiO}_{2}$ aerogel composites reinforced by SiC-coated mullite fibers for thermal insulations. Ceramics International, 2015, 41(1): 437-442.

[53] YOO J, BANG Y, HAN S J, et al. Hydrogen production by steam reforming of liquefied natural gas (LNG) over magnesium-doped nickel-alumina aerogel catalyst. Journal of Nanoscience and Nanotechnology, 2016, 16(10): 10835-10840.

[54] HAO Z, ZHU Q, JIANG Z, et al. Characterization of aerogel $\mathrm{Ni} / \mathrm{Al}_{2} \mathrm{O}_{3}$ catalysts and investigation on their stability for $\mathrm{CH}_{4}-\mathrm{CO}_{2}$ reforming in a fluidized bed. Fuel Processing Technology, 2009, 90(1): 113-121.

[55] CHEN JI-XIANG, WANG RI-JIE, LI YU-MIN, et al. Study of nickel-based aerogel catalyzing $\mathrm{CO}_{2}$ reforming of $\mathrm{CH}_{4}$ to synthesis gas. Journal of Fuel Chemistry and Technology, 2002, 30(1): 45-48.

[56] MI YU-HONG, ZHANG XIAO-BIN, LUO JUN-HANG, et al. Influence of calcining temperature of aerogel catalyst on its catalytic activity for synthesizing SWNTs. Journal of Inorganic Materials, 2007, 22(4): 604-608.

[57] GAO QING-FU, ZHANG CHANG-RUI, FENG JIAN, et al. Preparation and thermal performance of alumina aerogel insulation composites. Journal of National University of Denfense Technology, 2008, 30(4): 39-42.

[58] JIANG YING-YING, ZHANG LIN-PING, XU HONG, et al. Preparation and characterization of thermal protective aluminum hydroxide aerogel/PSA fabric composites. Journal of Sol-Gel Science and Technology, 2017, 82(2): 370-379.

[59] XIE HONG-YAN, YANG WEI, YUEN ANTHONY CHUN YIN, et al. Study on flame retarded flexible polyurethane foam/alumina aerogel composites with improved fire safety. Chemical Engineering Journal, 2017, 311: 310-317. 